\title{
ESNR Presidential Address, 2017
}

\begin{abstract}
Alex Rovira ${ }^{1}$
(C) Springer-Verlag Berlin Heidelberg 2016
\end{abstract}

I am honoured to become ESNR's 19th president, 47 years after the naming of its first President, Dr. George B. Ziedses des Plantes, and 12 years since another Spanish neuroradiologist, Dr. Jordi Ruscalleda, occupied this position.

Assuming the presidency of ESNR is certainly a huge responsibility and a challenging task, and despite being a bit nervous, I am excited and full of enthusiasm for the job ahead. The work of my predecessors, the creative input and energy of my colleagues on the Executive Committee, and the professionalism and efficiency of the ESNR staff at the Central Office will clearly facilitate my task.

ESNR is in good health. Membership in our society has grown from 315 paying members 10 years ago to almost 1300 individual members today. Moreover, more than 2800 institutional members from 25 countries are now represented in the ESNR Institutional Members Council. The Society's financial situation is also quite sound, enabling ESNR to promote educational, scientific, and research activities among our members.

The excellent educational and certification programmes provided by ESNR are one reason for the exponential increase in the membership to our Society. In fact, our postgraduate educational programme in diagnostic, paediatric, spinal, and interventional neuroradiology is probably the best in radiology and the envy of other European radiological societies. I believe this may be the most important achievement of the Society in the last few years, and I must acknowledge the keen vision of Professor Marco Leonardi as the founder of this great programme.

Alex Rovira

alex.rovira@idi.gencat.cat

1 Section of Neuroradiology (Department of Radiology), University Hospital Vall d'Hebron, Barcelona, Spain
Within the educational programme, the European Course of Neuroradiology (ECNR) is our most important activity. The 14th cycle of ECNR, under the Chairpersonship of Majda Thurner and Johan van Goethem, was initiated in October 2017, in Dubrovnik (Croatia). More than 320 students from 51 countries (88 students from 23 non-European countries) attended the first module of this 14th cycle, reflecting the high scientific level and excellent organization of the ECNR.

The ESNR has also been active in other areas. In 2008, thanks to the efforts of many members of the Society, the Division of Neuroradiology was created at the UEMS (Union Européenne des Médecins Spécialistes). Without a doubt, this achievement reflects the recognition of neuroradiology as a radiological subspecialty with specific and well-defined characteristics. One of the objectives of my presidency is to improve our relations with the UEMS Division of Neuroradiology, as this will facilitate homogenization of neuroradiology training and practice across Europe, in both diagnostic and interventional areas. More recently, ESNR created the European Board of Neuroradiology (EBNR), presently chaired by Professor Turgut Tali, which has its core activity organizing examinations that lead to certificates and diplomas in the field of neuroradiology. The vital mission of EBNR is to establish the European Diploma in Neuroradiology as an internationally recognized certificate of neuroradiological excellence.

We have also strengthened our relations with other societies, and in particular with the European Society of Radiology (ESR), whose current president, Professor Paul Parizel, is a neuroradiologist and past president of ESNR. We are collaborating with ESR in several educational, scientific, and professional activities with the main purposes of defending neuroradiology, both diagnostic and interventional, as a unified radiological subspecialty, and in promoting education and research in our field.

ESNR has been also working to increase our relations with ESMINT (European Society for Minimally Invasive 
Neurological Therapy), and I am fully convinced that we will achieve an agreement with this and other neuroscientific societies regarding an important issue; achieving excellence in the training and practice of interventional neuroradiology in Europe. This is also one of the main objectives of my presiden$\mathrm{cy}$, as it will enable the establishment of adequate training programmes in this line that will have clear benefits for patients.

Our current relationships with other scientific societies, such as the American Society of Neuroradiology (ASNR), American Society of Spine Radiology (ASSR), American Society for Paediatric Neuroradiology (ASPNR), and European Society of Head and Neck Radiology (ESHNR), are focused on learning from each other. To facilitate this goal, we are organizing joint sessions with some of these societies in our annual congresses. Recently, we created a Latin American-European Course of Neuroradiology (LATAM-ECNR) in collaboration with the Sociedade Paulista de Radiologia, which will be organized in Sao Paolo (Brazil) over the next 4 years. The objective of these efforts by ESNR is to promote neuroradiology across Europe and in other areas of the world and to take the lead in education, science, and the professional issues that affect our specialty.

The most important activity of our society is our ESNR Annual Congress. Another important task of my presidency is to increase the attendance and scientific level of this meeting and to improve its organization. To accomplish this, we have established new, more transparent rules for organizing the Congress, which have the purpose of objectively selecting the most appropriate venue and congress president, and providing a highly attractive scientific programme.

This coming year, the ESNR Annual Congress will take place in Malmo, Sweden (13-17 September 2017), under the presidency of Professor Pia Sundgren. Professor Sundgen and the members of the ESNR Scientific Programme Committee have worked hard to provide a compelling programme that can be viewed on our website. This programme, which includes high-quality scientific lectures as well as refresher and educational talks, will cover topics such as clinical-radiological correlations in movement disorders, neuroinflammation, an update on brain tumour imaging, imaging in psychiatric disorders, new advanced diffusion and perfusion imaging techniques, infectious disease, advanced spine imaging, paediatric sessions focusing on emergency situations, head and neck sessions, and many more. The problems related to standardising advanced imaging sequences, imaging protocols, and scanner performance will also be discussed. Joint sessions with other societies (e.g. ASNR, ASPNR, and ESHNR) have also been included in the programme. An Advanced Diagnostic Course related to neurodegenerative diseases is planned, including talks on multiple sclerosis, dementia, and movement disorders, as well as a keynote lecture on small vessel disease, while the Advanced Interventional Course will be an update on stroke imaging. For the first time, the Congress will begin on a Wednesday afternoon (13th September) with a half-day joint diagnostic and interventional session on idiopathic normal pressure hydrocephalus, including the latest news on the diagnosis and treatment of this condition. Several well-known international speakers have accepted our invitation to participate and will present keynote lectures and talks in the educational programme. The invited faculty includes Professors Anne Osborn, Mauricio Castillo, Walter Kucharczyk, Thomas Chenevert, and Leonardo Mandro, among others.

I believe the organizers have succeeded in putting together an excellent scientific programme and I expect a high attendance, not only of ESNR members but also of other specialists interested in neuroradiology.

Finally, I would like to comment briefly on our official journal, Neuroradiology. Professor Guido Wilms has been the Editor-in-Chief for the last 6 years, and I would like to thank him for his excellent work, in particular at this difficult time when there is a great deal of competition from other journals and other types of publishing resources. At the same time, I welcome the new Editor-in-Chief, Professor Rüdiger von Kummer, a well-known neuroradiologist with an outstanding scientific background and previous experience in editorial tasks. One of the objectives of the new Editor-in-Chief is to make Neuroradiology a real challenger to other neuroradiological journals by increasing the number of editorials on hot topics, pro and con debates, and state-of-the-art reviews. By continually alerting the radiologic and neuroscience community to new publications in Neuroradiology, the visibility of our official journal will improve. The final goal is to increase the scientific quality of the journal, attracting young and senior neuroradiologists from all over the world to publish their research there. We cannot ignore that a significant percentage of the research currently performed in neuroradiology is led by professionals other than neuroradiologists and published in non-radiological journals. The only way to reverse this situation is to promote research activities among our members and increase the quality of our Journal and Annual Congress.

I cannot end without recognizing the excellent work done by all the ESNR presidents. It has been a great pleasure to work with them over the last 13 years as an Executive Committee member. All of them, with their unique profiles and styles, have made important contributions to the Society, and I have learned a lot from them.

Let me conclude this presidential address by inviting all ESNR members to participate in our activities and in the organization of our Society. It will be a pleasure for me and for all ESNR executive members to receive any suggestions you consider relevant.

With your support and active participation, I am fully convinced that we will be able to further improve our already successful Society.

I wish you all the best for 2017 .

Alex Rovira Cañellas

President, ESNR 